\title{
A Novel DOA Measurement Approach for Coherent Signals In MIMO-OFDM System
}

\author{
Huanghe $\mathrm{LI}^{1 \text {, a }}$, Xiaoyou $\mathrm{YU}^{1, \mathrm{~b}^{*}}$, Heng $\mathrm{YE}^{1}$, Yan $\mathrm{ZHOU}^{1}$, Nengda $\mathrm{Cl}^{1}$, \\ Pengshuai SUN ${ }^{1}$, Zhinian LUO ${ }^{1}$ \\ ${ }^{1}$ Department of Communication Engineering, College of Computer Science and Electronic \\ Engineering, Hunan University, Changsha 410082, China
}

aemail: 295679756 @qq.com, bemail: yu.xiaoyou@163.com

Keywords: Vehicle Environment; MIMO-OFDM; DOA; Coherent Signals

\begin{abstract}
In order to solve the problem of low spatial resolution caused by signal correlation for angle estimation, a novel DOA measurement approach for related signal is proposed based on vehicle MIMO-OFDM signal. The proposed method uses the cross-correlation matrix to reconstruct the signal covariance matrix, which can simultaneously estimate the DOAs of relevant and irrelevant signals. Simulation results indicate that the proposed method provides higher resolution performance for coherent sources compared to spatial smoothing algorithms, and the computational complexity is also reduced to some extent.
\end{abstract}

\section{Introduction}

Since the concept of intelligent transportation systems (ITS) is put forward, research for Internet of Vehicles (IOV) [1-2] has become more widely. As a key research area of ITS, IOV system can not only realize Vehicles-to-vehicles(VTV) and road-to-vehicles(RTV) communciation,but also have the ability of radar sensing. In [3],the Orthogonal frequency division multiplexing (OFDM) radar-communication integration system (RadCom) is proposed. IEEE 802.11p standard for IOV system also employs OFDM signal[4].For IOV system,the range-velocity estimate algorithms has been proposed[5] and verified[6]. However, in order to achieve the functions of localization, it is necessary to measure the angular orientation of the target.

In wireless communication system, multiple input multiple output (MIMO) system can greatly increase the channel capacity; OFDM technique has strong robustness against fading and multipath propagation effects,and with high spectral efficiency. Therefore ,the combination of MIMO and OFDM is conducive to accurately estimate the angle. However, in vehicle environment, due to the presence of various reflector and scatterer, it is likely to cause the receiver signal correlated.

Many methods have been proposed to solve the problem of DOA estmation for coherent signals, such as the spatial smoothing technique, singular value decomposition (SVD), Toeplitz algorithms[7], etc. The most commonly used is spatial smoothing technique [8]. In order to reduce the number of the sensors, an improved smoothing algorithm is proposed, which referred to as the forward/backward spatial smoothing (FBSS) algorithm[9].

However, these algorithms have poor performance at low SNR and high computational complexity. To improve the performance,in this paper, a novel high-resolution DOA measurement approach for coherent signals is proposed for automotive environment.

\section{MIMO-OFDM System Model}

Consider MIMO-OFDM system with $N_{t}$ transmit antennas and $N_{r}$ receive antennas in Fig.1. The antenna arrays are uniformly spaced linear array,and are spaced apart by $d_{r}$ at the receiver. In this paper, $d_{r}=\lambda / 2$, where $\lambda$ is the carrier wavelength.

At the receiving end, the processing of the received signal involves the transformation of a two-dimensional array of $N_{t} \times N_{r}$ into one-dimensional pseudo antenna array of $1 \times \mathrm{P}$, where 
$\mathrm{P}=N_{t} \times N_{r}$.The details of the transformation have been described in [10]、 [11].

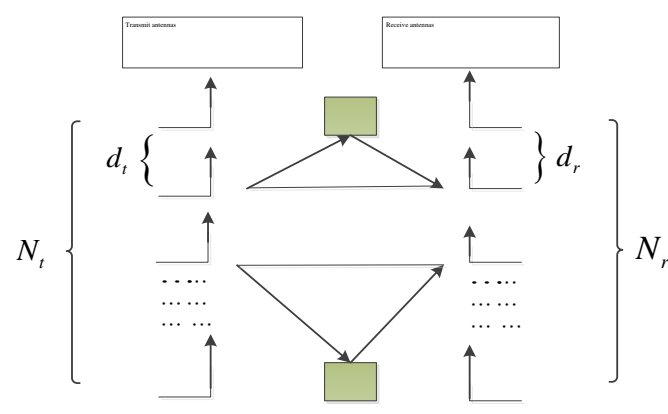

Fig.1. MIMO antenna array

Assuming that each OFDM symbol contains $N$ subcarriers. One frame data includes $L$ OFDM symbols. In this paper ,an interleaving frame structure is employed. In the frame structure,selected subcarriers instead of consecutive OFDM subcarriers will be allocated to selected transmit antennas. With the interleaving structure, the time-domain signal transmitted at the i-th antenna can be expressed as:

$$
x_{i}(t)=\sum_{\mu=0}^{L-1} \sum_{\hat{n}=n N_{t}+i-1}^{N-1} D_{i}(\mu N+\hat{n}) \exp \left(j 2 \pi f_{\hat{n}} t\right) \operatorname{rect}\left(\frac{t-\mu T}{T}\right), n=0, \ldots, N-1
$$

where $f_{\hat{n}}$ represents the subcarrier frequency, $T$ denotes the OFDM symbol duration, $D(n)$ is the symbol after QPSK modulation. rect() represents rectangular pulse shaping function.

In the channel , when the transmited signal is reflected by an object at the distance of $R$, the received signals will produce a linear phase rotation of $\exp \left(-\mathrm{j} 2 \pi \mathrm{n} \Delta \mathrm{f} 2 R / c_{0}\right)$, where $c_{0}$ is the speed of light. The recived time-domain baseband signal at the receive anttena $m$ is given :

$$
y_{m}(t)=\sum_{i=1}^{N_{t}} \sum_{\mu=0}^{L-1} \sum_{n=0}^{N-1} D_{r_{i}}(\mu, \hat{n}) \exp \left(j 2 \pi f_{\hat{n}} t\right)+w(t), m=1, \ldots, N_{r}
$$

where $w(t)$ is the Additive Gaussian White Noise. $D_{r_{i}}(\mu, \hat{n})$ represents the complex modulation symbol,which can be expressed as $D_{r_{i}}(\mu, \hat{n})=D_{i}(\mu, \hat{n}) \exp \left(-j 2 \pi f_{\hat{n}} 2 R_{i} / c_{0}\right)$.

The time-variant channel transfer function can be obtained from comparing the received symbol $D_{r}(\mu, n)$ and the transmitted symbol $D(\mu, n)$. This is computed by an element-wise division:

$$
D_{\text {div }}(\mu, n)=\frac{D_{r}(\mu, n)}{D(\mu, n)}
$$

After inverse discrete Fourier transform (IDFT),the transfer function can be expressed as:

$$
r(k)=\operatorname{IDFT}\left(\left\{D_{\text {div }}(\mu, n)\right\}\right)=\frac{1}{N} \sum_{n=0}^{N-1} \exp \left(-j 2 \pi n \Delta f \frac{2 R}{c_{0}}\right) \exp \left(j 2 \pi \frac{n}{N} k\right), k=1, \ldots N
$$

\section{Correlation Analysis of the Received Signal}

In vehicle environment, since the presence of the multi-path effects, it is likely to cause the receiver signal correlated. Assuming that there are $M$ narrowband signals arrived at the receiver.The DOAs , respectively, were $\theta_{1}, \theta_{2}, \ldots, \theta_{M}$. The input for the estmation algorithm is:

$$
\mathbf{S}(k)=\left[r_{1}(k), r_{2}(k), r_{3}(k), \ldots, r_{M}(k)\right]^{T}
$$

The interval of the pseudo receive antenna is $d_{r_{i}}=\left(p_{i}-1\right) \lambda / 2$, where $p_{i}=1, \ldots, N_{t} N_{r}$. The array manifold vector is $\mathbf{A}(\theta)=\left[\boldsymbol{\alpha}\left(\theta_{1}\right), \boldsymbol{\alpha}\left(\theta_{2}\right), \ldots, \boldsymbol{\alpha}\left(\theta_{M}\right)\right]$, where $\quad \boldsymbol{\alpha}\left(\theta_{i}\right)=\left[1, e^{-j \omega_{i}}, \ldots, e^{-j(P-1) \omega_{i}}\right]^{T}$, $\omega_{i}=2 \pi \sin \left(\theta_{i}\right) d_{r} / \lambda$.

Taking snapshot at time $k=1,2, \ldots, N$, the output of $P$ elements $\mathbf{Y}(k)$ can be expressed 
as : $\mathbf{Y}(k)=\sum_{i=1}^{M} \boldsymbol{\alpha}\left(\theta_{i}\right) * \mathbf{r}_{i}(k)+\mathbf{W}(k) . \mathbf{W}(k)$ is the Additive Gaussian White Noise . The covariance matrix of $\mathbf{Y}(k)$ is given:

$$
\mathbf{R}_{y}=E\left\{\mathbf{Y}(k) \mathbf{Y}^{H}(k)\right\}=\mathbf{A T A} \mathbf{A}^{H}+\sigma^{2} \mathbf{I} .
$$

Where $\mathbf{T}=E\left\{\mathbf{S}(k) \mathbf{S}^{H}(k)\right\}$; If $r_{i}(k)$ and $r_{j}(k)$ are related, $i, j=1, \ldots, M$, the matrix $\mathbf{T}$ will be no longer diagonal matrix.If $\mathbf{T}$ is not diagonal matrix, $\mathbf{R}_{y}$ will not be full rank matrix. The commonly used algorithms,such as MUSIC algorithm, will not be able to accurately estimate the DOAs .

\section{DOA Estimation Method for Coherent signal}

In this paper, an novel DOA estimation method for coherent signal is proposed to improve the performance. Firstly, the covariance matrix of $\mathbf{Y}(k)$ can be written as:

$$
\mathbf{R}_{y}=E\left\{\mathbf{Y}(k) \mathbf{Y}^{H}(k)\right\}=\frac{1}{N} \sum_{k=1}^{N} \mathbf{Y}(k) \mathbf{Y}^{H}(k)
$$

In order to construct a new covariance matrix, a reverse unit matrix $\mathbf{G}$ is introduced. With the matrix $\mathbf{G}$, construct a new matrix $\mathbf{Z}(k)=\mathbf{G} \mathbf{Y}^{*}(k)$, where $\mathbf{Y}^{*}(k)$ is the complex conjugate matrix of $\mathbf{Y}(k)$.The covariance matrix of $\mathbf{Z}(k)$ can be expressed as:

$$
\mathbf{R}_{Z}=E\left\{\mathbf{Z Z} \mathbf{Z}^{H}\right\}=E\left[\mathbf{G} \mathbf{Y}^{*}(k)\left(\mathbf{G} \mathbf{Y}^{*}(k)\right)^{H}\right]=\mathbf{G R}_{y}^{*} \mathbf{G}
$$

The cross correlation matrix of $\mathbf{Y}(k)$ and $\mathbf{Z}(k)$ is $\mathbf{R}_{y z}=E\left\{\mathbf{Z} \mathbf{Y}^{H}\right\}$.Construct two novel matrixs: $\mathbf{R}_{1}=\mathbf{R}_{y}+\mathbf{G} \mathbf{R}_{y z}{ }^{*} \mathbf{G}$, and $\mathbf{R}_{2}=\mathbf{R}_{z}+\mathbf{G} \mathbf{R}_{y z}{ }^{*} \mathbf{G}$.A new covariance matrix is given :

$$
\mathbf{R}=\frac{\mathbf{R}_{1}+\mathbf{R}_{2}}{2}=\frac{\mathbf{R}_{y}+\mathbf{R}_{z}+2 \mathbf{G} \mathbf{R}_{y z}^{*} \mathbf{G}}{2}
$$

According to the matrix theory, $\mathbf{R}$ is a full rank matrix.With the new covariance matrix $\mathbf{R}$, the DOAs can be achieved with MUSIC algorithm.

\section{Simulation Results}

Assuming OFDM signals are transmitted and received with 4X4 MIMO. The resulting number of pseudo receive antenna is thus ,16.The antennas are spaced a half wavelength.The OFDM parameters are set according to the IEEE 802.11p standard. Assuming that the noise is Gaussian white noise, the number of snapshots is $64 * 64$,and there are 5 signals arrived in the array with the angles of $-45^{\circ},-12^{\circ},-10^{\circ}, 10^{\circ}, 11^{\circ}$. The signal 2 and signal 3 are coherent sources,and the signal 4 and signal 5 are correlated to each other. The signal 1 is independent to others. SNR=10db. Fig. 3 shows the DOA performance of spatial smoothing algorithm and the proposed method.

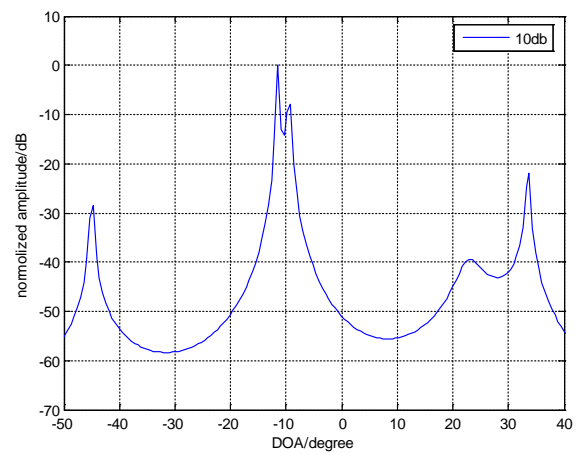

(a) DOA performance of Forward/Backward spatial smoothing algorithm

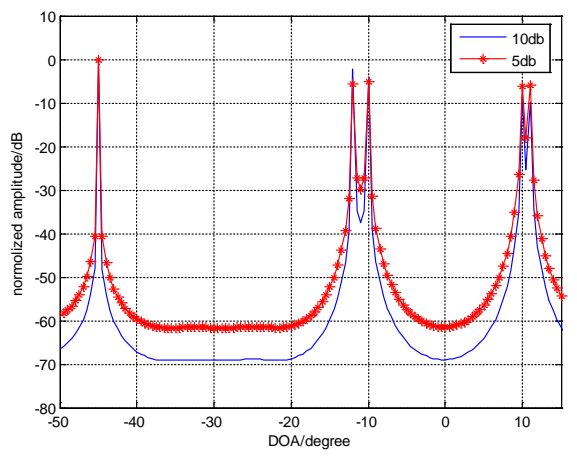

(b) DOA performance of the proposed method

Fig.2. The experimental results

As is shown in the figure, the spatial smoothing algorithm can not estimate all the DOAs in 
condition of $\mathrm{SNR}=10 \mathrm{~dB}$ when relevant and irrelevant signals simultaneously exist. However,the proposed method can simultaneously estimate all the DOAs. The peaks are also very sharp even when the SNR is $5 \mathrm{~dB}$. And the proposed can distinguish two coherent signals which spaced $1^{\circ}$. Therefore,the performance of the proposed method is superior to spatial smoothing algorithm.

On the other hand, since the proposed method do not need to deal with multiple sub-array signal, it requires substantially lower computations. Therefore, compared to the spatial smoothing algorithm ,the proposed estmation method is more suitable for vehicle applications.

\section{Conclusion}

In vehicle environment, since the carrier frequency of transmitted signal is equal and the presence of multi-path effects,it is likely to cause the receiver signal correlated. In order to solve the problem of low spatial resolution caused by signal correlation for angle estimation, a novel DOA measurement approach for related signal is proposed for MIMO-OFDM system in vehicle environment. Numerical simulations show that the proposed method provides higher resolution performance for coherent sources compared to conventional spatial smoothing algorithms, which can distinguish the correlation signal which spaced $1^{\circ}$,and more suitable for vehicle applications.

\section{Acknowledgement}

In this paper, the research was sponsored by the National Natural Science Foundation of China (Project No. 61371115).

\section{References}

[1]Kim K, Lee J, Lee W. A MAC Protocol Using Road Traffic Estimation for Infrastructure-to-Vehicle Communications on Highways[J]. IEEE Transactions on Intelligent Transportation Systems, 2013, 14(14):1500-1509.

[2] Mecklenbrauker C F, Molisch A F, Karedal J, et al. Vehicular Channel Characterization and Its Implications for Wireless System Design and Performance[J]. Proceedings of the IEEE, 2011, 99(7):1189-1212.

[3] Sturm C, Zwick T, Wiesbeck W. An OFDM System Concept for Joint Radar and Communications Operations[C]. Vehicular Technology Conference, 2009. VTC Spring 2009. IEEE 69th. IEEE, 2009:1 - 5.

[4] Reichardt, L, Sturm, C, Grunhaupt, F, et al. Demonstrating the use of the IEEE 802.11P Car-to-Car communication standard for automotive radar[C].European Conference on Antennas and Propagation. IEEE, 2012:1576-1580.

[5] Sturm C, Braun M, Zwick T, et al. A multiple target doppler estimation algorithm for OFDM based intelligent radar systems[C]. Radar Conference. IEEE, 2010:73-76.

[6] Sturm C, Zwick T, Wiesbeck W, et al. Performance verification of symbol-based OFDM radar processing[J]. 2010, 29(16):60-63.

[7] Merchant G, Parks T W. Efficient solution of a Toeplitz-plus-Hankel coefficient matrix system of equations[J]. IEEE Transactions on Acoustics Speech \& Signal Processing, 1982, 30(1):40-44.

[8] Shan T J, Wax M, Kailath T. On spatial smoothing for direction-of-arrival estimation of coherent signals[J]. IEEE Transactions on Acoustics Speech \& Signal Processing, 1985, 33(4):806-811.

[9] Pillai S U, Kwon B H. Forward/Backward spatial smoothing techniques for coherent signal Identification[J]. IEEE Transactions on Acoustics Speech \& Signal Processing, 1989, 37(1):8-15. 
[10] Schuler K. Intelligente Antennensysteme für Kraftfahrzeug-Nahbereichs-Radar-Sensorik[J].

[11] Bliss D W, Forsythe K W. Multiple-input multiple-output (MIMO) radar and imaging: degrees of freedom and resolution[C]. Signals, Systems and Computers, 2004. Conference Record of the Thirty-Seventh Asilomar Conference on. IEEE, 2003:54-59 Vol.1. 